quantities are not large and then insensitive to uncertainties in the calculations.

The above numbers are relevant to the subclass of decays selected by the specific character of the experimental design and a more universal expression of the results is necessary. For the kinematic region such that $\overrightarrow{\mathrm{p}}_{\mu} \cdot \overrightarrow{\mathrm{p}}_{v}=0$, we have $P_{n}$ $=(-3.0 \pm 4.7) \times 10^{-3}$. We can also express the result in terms of the value of $\xi$, the conventional ratio of form factors used in discussion of $K_{\mu 3}$ decays; taking $\operatorname{Re} \xi=0$, we find $\operatorname{Im} \xi=-0.016$ \pm 0.025 .

According to the calculations of Zhitnitskii, $C P$ nonconservation in the Higgs sector leads to a polarization, for $P_{n}$ where $\overrightarrow{\mathrm{p}}_{\mu} \cdot \overrightarrow{\mathrm{p}}_{v}=0$ and $T_{\mu}$ is of the order of $100 \mathrm{MeV}$, such that

$$
P_{n}=\frac{\left(m_{\mu} m_{K}\right)}{\left(8 m_{0}^{2}\right)^{1 / 2}} \frac{v_{2}^{2}}{v_{3}^{2}}=-4.63 \times 10^{-3} \frac{v_{2}^{2}}{v_{3}^{2}},
$$

where the value of $m_{0}$ is set equal to $2 \mathrm{GeV}$ by the measured $K^{0}-\bar{K}^{0} C P$-nonconserving effects and $v_{2}^{2} / v_{3}^{2}$ is defined by the gauge transformation properties of the Higgs doublets and not otherwise determined experimentally: Zhitnitskii expects this number to be of the order of one. For this experiment, the limits set are of the magnitude expected and serve to constrain the free parameter to be less than two.

Somewhat stronger constraints can be invoked if we compound these results with those from our previous measurements ${ }^{6}$ of $P_{n}$ from the $K_{\mu_{3}}$ decays of $K_{L}{ }^{0}$ mesons where we found $\operatorname{Im} \xi=0.001$ \pm 0.030 . The experiments are quite similar and the uncertainties are almost wholly statistical. Therefore, we feel that it is permissible to add the results of the two measurements to derive a value of $\operatorname{Im} \xi=-0.010 \pm 0.019$ and $P_{n}=(-1.85$ $\pm 3.60) \times 10^{-3}$. Although this null result cannot exclude the possibility that the $C P$-nonconserving effects which are known from observations of the $K^{0}-\bar{K}^{0}$ system derive from $C P$-nonconserving phases in the Higgs sector, we can conclude that such mechanisms do not make unusually large contributions to $K_{\mu_{3}}$ decays.

This work was supported by the U. S. Department of Energy.

${ }^{1}$ J. H. Christenson, J. W. Cronin, V. L. Fitch, and R. Turlay, Phys. Rev. Lett. 13, 138 (1964).

${ }^{2}$ M. Kobayashi and K. Maskawa, Prog. Theor. Phys. 49,652 (1973).

${ }^{3}$ S. Weinberg, Phys. Rev. Lett. 37, 657 (1976).

${ }^{4}$ A. R. Zhitnitskii, Yad. Fiz. 31, 1024 (1980) [Sov. J. Nucl. Phys. 31, 529 (1.981)]; however, calculations by A. I. Sanda, Phys. Rev. D $\underline{23}, 2647$ (1981), and by N. G. Deshpande, Phys. Rev. D 23 , 2654 (1981), suggest that such Higgs models may lead to unacceptably large values of $\epsilon^{\prime}$.

${ }^{5}$ L. B. Leipuner et al., IEEE Trans. Nucl. Sci. $\underline{28}$, 333 (1981).

${ }^{6} \mathrm{~W}$. M. Morse et al., Phys. Rev. D $\underline{21}, 1750$ (1980).

\title{
Superstrong Force with a Heavy Axion
}

\author{
S. -H. H. Tye \\ Newman Laboratory of Nuclear Studies, Cornell University, Ithaca, New York 14853
} (Received 17 April 1981)

\begin{abstract}
A new, superstrong force (analogous, to, but much stronger than, quantum chromodynamics) is introduced to the Peccei-Quinn extension of the standard model of weak and electromagnetic interactions. Strong $C P$ nonconservation is naturally suppressed and the resulting heavy axion is compatible with the present experimental bounds. The strength of this new force and some of its properties are discussed.
\end{abstract}

PACS numbers: $12.20 . \mathrm{Hx}, 14.80 . \mathrm{Kx}$

The discovery of pseudoparticle solutions ${ }^{1}$ in non-Abelian gauge theories has far-reaching consequences in quantum chromodynamics (QCD). The $Q C D$ vacuum must have a nontrivial topological structure, ${ }^{2}$ labeled by a new parameter $\theta_{c}$, and the effective QCD Lagrangian becomes

$$
L_{\mathrm{eff}}=L_{\mathrm{QCD}}+\left(i \theta_{c} g^{2} / 32 \pi^{2}\right) G_{\mu \nu}{ }^{a} \tilde{G}^{a \mu \nu},
$$

where $\tilde{G}^{a \mu \nu}$ is the dual of the QCD field stress tensor $G^{a \mu \nu}$ and $g$ is the QCD coupling constant. The presence of the second term violates $P$ and $C P$ invariances, both of which are known to be excellent symmetries of strong interactions. In fact, the experimental bound on the electric dipole moment of the neutron ${ }^{3}$ implies $\theta_{c}<10^{-8}$. This means that $\theta_{c}$ must be extremely small if not exactly zero. It is possible, although highly unlikely, that nature just so happens to have a 
very tiny $\theta_{c}$. Furthermore, there are no experiments that can be performed to find out if nature has made this accidental choice. It seems more likely that $\theta_{c}=0$ in nature because this is dictated by the presence of some symmetry.

One way to achieve this is to have a new global chiral U(1) symmetry on the QCD Lagrangian. Invariance under this chiral rotation renders theories with different $\theta$ values all equivalent to one another. Then it follows that the effective $\theta$, namely $\theta_{c}$, is exactly zero. There are at least two ways to realize this $U(1)$ symmetry. One way is to have a massless quark, the $u$ quark being the only possible candidate. Unfortunately, if the $u$ quark is massless, our present understanding of current algebra must be modified somewhat in order to be compatible with experiments. Again, in this case, there are no experiments that can be performed to test directly the masslessness of the $u$ quark.

The other way to realize this global U(1) symmetry is that suggested by Peccei and Quinn ${ }^{4}$ [to be called $\left.\mathrm{U}(1)_{\mathrm{PQ}}\right]$. The simplest version of a realistic model that contains this $\mathrm{U}(1)_{\mathrm{PQ}}$ symmetry combines QCD with the standard weakelectromagnetic interaction model $^{5}$ which contains two Higgs doublets. Weinberg ${ }^{6}$ and Wilczek observed that, as a result of $\mathrm{U}(1)_{\mathrm{PQ}}$, a light pseudoscalar Higgs boson (the axion) must be present. Unfortunately, such an axion (with a typical mass around $100 \mathrm{keV}$ ) seems to have been rules out by experiments. In fact, the experimental bound on the mass of such an axion is roughly $m_{a} \approx 50$ $\mathrm{MeV}{ }^{8}$ A number of modifications of this model have been suggested by various authors so that the resulting modified models satisfy the present experimental bounds on the axion. ${ }^{9}$ Here I propose a new model for the axion by extending the Peccei-Quinn model to include a new, as yet unobserved, superstrong interaction. The new interaction (to be called $\mathrm{QC}^{\prime} \mathrm{D}$ ) raises the axion mass by a huge factor so that the axion's existence is compatible with the present experimental bound. Here I shall consider only a simple version of this new force, whose properties can be extrapolated from QCD. This new force offers many interesting properties and dramatic signatures for detection, some of which I shall briefly discuss.

The Lagrangian is given by

$$
L=L_{\mathrm{QCD}}+L_{\mathrm{WS}}+L_{\mathrm{QC}}+L_{\mathrm{Yuka} \text { wa }}-V\left(\varphi_{1}, \varphi_{2}\right),
$$

where $L_{\text {WS }}$ is the standard Weinberg-Salam-Glashow-Iliopoulos-Maiani model. The new interaction $Q C^{\prime} D$ has its own fermions which are in the fundamental representations of a gauge group $G$ and are singlets under the QCD color SU(3) group. The gauge group $G$ is unbroken and its fermions are confined at a distance scale much smaller than that in QCD. The $\mathrm{QC}^{\prime} \mathrm{D}$ fermions are lefthanded doublets and right-handed singlets under the weak $\mathrm{SU}(2)$ gauge group, to be called $\left(P_{i}, N_{i}\right)$, just like the ordinary quark doublets $\left(\boldsymbol{p}_{j}, n_{j}\right)$, where $i$ and $j$ are generation indices (capital letters to be used for $\mathrm{QC}$ 'D objects and small letters for $\mathrm{QCD})$, so that the lightest pair is $(U, D)$ in $Q C^{\prime} \mathrm{D}$ and is $(u, d)$ in $\mathrm{QCD}$. The Higgs potential is chosen such that spontaneous symmetry breaking takes place $\left(f^{2}=f_{1}{ }^{2}+f_{2}^{2}, x=f_{1} / f_{2}\right)$ :

$$
\begin{aligned}
& \varphi_{1}=\frac{f_{1}}{\sqrt{2}}\left(\begin{array}{l}
1 \\
0
\end{array}\right) \exp \left(\frac{i x a}{f}\right), \\
& \varphi_{2}=\frac{f_{2}}{\sqrt{2}}\left(\begin{array}{l}
0 \\
1
\end{array}\right) \exp \left(\frac{i a}{x f}\right),
\end{aligned}
$$

where $a$ is to be identified as the axion field. ${ }^{6,7}$ The Yukawa couplings are such that $\varphi_{1}\left(\varphi_{2}\right)$ gives masses to $P_{i}\left(N_{i}\right)$ and $p_{j}\left(n_{j}\right)$ so that the flavorchanging neutral current is naturally suppressed. Following Peccei and Quinn, the Lagrangian has an additional chiral $U(1)$ symmetry so that, after symmetry breaking, the axion part of the Lagrangian has the $\mathrm{U}(1)_{\mathrm{PQ}}$ symmetry explicit in it:

$$
\begin{aligned}
L_{a}= & \frac{1}{2}\left(\partial_{\mu} a\right)^{2}-\sum_{i} M_{P_{i}} \bar{P}_{i} \exp \left(i x a \gamma_{5} / f\right) P_{i}-\sum_{i} M_{N_{i}} \bar{N}_{i} \exp \left(i a \gamma_{5} / x f\right) N_{i} \\
& -\sum_{k} m_{l_{k}} \bar{l}_{k} \exp \left(i a \gamma_{5} / x f\right) l_{k}-\sum_{j} m_{p_{j}} \bar{p}_{j} \exp \left(i x a \gamma_{5} / f\right) p_{j}-\sum_{j} m_{n_{j}} \bar{n}_{j} \exp \left(i a \gamma_{5} / x f\right) n_{j},
\end{aligned}
$$

where the leptons $l_{k}$ are also included. The axion couplings to usual quarks, usual leptons, and $\mathrm{QC} \mathrm{C}^{\prime} \mathrm{D}$ fermions are determined by Eq. (4). The axial current associated with this residual chiral U(1) symmetry is

$$
J_{5 \mu}=f \partial_{\mu} a+\frac{1}{2} x\left(\sum \bar{P}_{i} \gamma_{\mu} \gamma_{5} P_{i}+\sum \bar{p}_{j} \gamma_{\mu} \gamma_{5} p_{j}\right)+(2 x)^{-1}\left(\sum \bar{N}_{i} \gamma_{\mu} \gamma_{5} N_{i}+\sum \bar{n}_{j} \gamma_{\mu} \gamma_{5} n_{j}+\sum \bar{l}_{k} \gamma_{\mu} \gamma_{5} l_{k}\right) .
$$

The divergence of this current is zero except for the QCD and the QC'D anomalies ${ }^{2,10}$

$$
\partial^{\mu} J_{5 \mu}=\left(1 / 32 \pi^{2}\right)\left(x+x^{-1}\right)\left(n g^{2} \tilde{G}_{\mu \nu}{ }^{a} G^{a \mu \nu}+N g^{\prime 2} \tilde{F}_{\mu \nu}{ }^{b} F^{b \mu \nu}\right),
$$


where $n(N)$ is the number of QCD (QC'D) doublets and $F_{\mu \nu}^{b}\left(\tilde{F}_{\mu \nu}^{b}\right)$ is the field stress tensor (its dual) of $Q C^{\prime} \mathrm{D}$. These anomalies actually render the axion massive. The $\mathrm{QC}^{\prime} \mathrm{D}$ coupling $g^{\prime}$ is taken to be much larger than the QCD coupling $g$ and, as we shall see below, the $U$ and $D$ masses $M_{U, D}$ are taken to be much larger than the $u, d$ masses $m_{u, d}$. Hence the $\mathrm{QC} C^{\prime} \mathrm{D}$ anomaly provides the dominant contribution to the axion mass and it is a good approximation to ignore the QCD anomaly contribution in Eq. (6).

To obtain some idea of the $\mathrm{QC}^{\prime} \mathrm{D}$ contribution to the axion mass, let us introduce the scale parameter $\Lambda_{c}$, in $\mathrm{QC}^{\prime} \mathrm{D}$. The corresponding scale parameter in QCD has the value $\Lambda_{c} \sim 0.2 \mathrm{GeV}$. If $M_{U, D} \ll \Lambda_{c^{\prime}}$, then the axion mass is dictated by the chiral symmetry limit and the standard current-algebra method can be applied. ${ }^{10}$ In general, both the anomaly and the chiral symmetry breaking by the fermion masses are important. With use of a $\mathrm{U}(2)$ nonlinear sigma model involving the $\mathrm{QC}$ 'D pions and “ $\eta$ " meson with an explicit $\mathrm{U}(1)$-symmetry-breaking interaction, ${ }^{10}$ the axion mass is given by (assuming $N=1$ )

$$
f^{2} m_{a}^{2} \simeq F_{\pi}^{2} M_{\pi}^{2}\left(M_{\eta}^{2}-M_{\pi}^{2}\right) / M_{\eta}^{2},
$$

where $\pi$ and $\eta$ are the isospin triplet and singlet pseudoscalar mesons in $\mathrm{QC}^{\prime} \mathrm{D}$. For simplicity, $Z=M_{U} / M_{D} \simeq 1$ and $x \simeq 1$. Scaling from $\mathrm{QCD}$, the QC'D pion decay constant is $F_{\pi} \simeq \frac{1}{2} \Lambda_{c}$, and the $\mathrm{U}(1)$ breaking due to the anomaly is $M_{\eta}{ }^{2}-M_{\pi}{ }^{2}$ $\simeq 7 \Lambda_{c}{ }^{2}$. As is clear from Eq. (7), if $\Lambda_{c}, \gg M_{U}$, the axion mass is obtained by using current-algebra methods, ${ }^{10}$

$$
\begin{aligned}
f^{2} m_{a}^{2} \simeq\left(x+\frac{1}{x}\right)^{2} \frac{Z}{(1+Z)^{2}} & F_{\pi}^{2} M_{\pi}^{2} \\
& \simeq M_{U} V_{U}+M_{D} V_{D},
\end{aligned}
$$

where $V_{U} \simeq V_{D} \simeq\left\langle 0\left|\bar{U} \exp \left(i a \gamma_{5} / f\right) U\right| 0\right\rangle \simeq \Lambda_{c},{ }^{3}$. If $M_{U} \gg \Lambda_{c^{\prime}}$, the meson mass is the sum of the fermion mass, the antifermion mass, and the binding energy; using our experience from QCD and intuitive dimensional arguments, I use $M_{\pi} \simeq 2\left(M_{U}\right.$ $\left.+\Lambda_{c^{\prime}}\right)$ as an order-of-magnitude estimate. This completes the estimate of the axion mass in terms of $M_{\pi}$ and $\Lambda_{c^{\prime}}$. Given $M_{\pi}$ and $\Lambda_{c^{\prime}}$, properties of $\mathrm{QC}^{\prime} \mathrm{D}$ are essentially determined. We shall come back to this later.

In the model described above, there are two $\theta$ angles which may induce large $C P$-nonconserving effects, namely $\theta_{c}$ in $\mathrm{QCD}$ and $\theta_{c^{\prime}}$ in $\mathrm{QC} \mathrm{C}^{\prime} \mathrm{D}$. The $\mathrm{U}(1)_{\mathrm{PQ}}$ symmetry allows us to make one rotation so that either the effective $\theta_{c}$ or the $\theta_{c}$ ( (or both) remains nonzero and $C P$ invariance remains bro- ken. Depending on the mass of $M_{U}$ (assuming $M_{U} \ll M_{D}$, it is possible to have $M_{U}<m_{u} \simeq 5 \mathrm{MeV}$ ), the $C P$ nonconservation may be suppressed somewhat. However, this is still far from sufficient in providing a natural suppression in the value of the electric dipole moment of the neutron. Also, the lightest $Q C^{\prime} D$ fermion is stable in this case, which may be difficult to reconcile with cosmological constraints. What is actually more likely to happen is that $\mathrm{QCD}$ and $\mathrm{QC} \mathrm{C}^{\prime} \mathrm{D}$ are unified into a bigger gauge group at some scale $M$ somewhere between $10^{2}$ and $10^{14} \mathrm{GeV}$. This implies that $\theta_{c}$ must be equal to $\theta_{c^{\prime}}$, at least at the tree level. Hence the possibility of a nonzero $\Delta \theta=\theta_{c^{\prime}}-\theta_{c}$ must come from radiative corrections. This implies that the neutron electric dipole moment can arise only from high-order-loop diagrams. Of course, the exact value depends on the details of the model. Experience from the Kobayashi-Maskawa model ${ }^{11}$ leads us to believe that radiative corrections at very high orders may be needed to induce a nonzero value of $\Delta \theta$. If this is the case, the neutron electric dipole moment induced by $\Delta \theta$ would be too small to be observed.

The above discussion leads us to consider the gauge group $G \otimes \mathrm{SU}(2)_{L} \otimes \mathrm{U}_{Y}(1)$ where the fermions either are spectators of the gauge group $G$ or have vectorlike interactions under $G$. (The fermion content must be chosen so that it is free of the weak-interaction anomalies.) The Higgs representations (e.g., two Higgs doublets) are chosen so that the Peccei-Quinn mechanism is in effect: $\bar{\theta}_{G}=\theta_{G}+\arg \operatorname{det} m=0$. The gauge group $G$ is broken into $\mathrm{QCD} \otimes \mathrm{QC} \mathrm{C}^{\prime} \mathrm{D}$ at the mass scale $M$. The Yukawa couplings of $\mathrm{QCD}$ quarks $(q)$ and $\mathrm{QC} \mathrm{C}^{\prime} \mathrm{D}$ fermions $(Q)$ are related since both $q$ and $Q$ come from fermion representations of $G$. For example, if $q_{i}$ and $Q_{i}$ with the same $\mathrm{SU}(2)_{L} \otimes \mathrm{U}_{Y}(1) \otimes$ family quantum numbers belong to the same irreducible representation of $G$, their "current quark" masses must come from the same Yukawa couplings, which guarantees that (i) at least at the tree level, the Yukawa couplings of each $\left(q_{i}, Q_{i}\right)$ pair to each of the two Higgs doublets have equal magnitude and phase, and (ii) $\bar{\theta}_{c}=\bar{\theta}_{c}^{\prime}=0$. Since $Q C^{\prime} D$ is a stronger force than $\mathrm{QCD}$, radiative corrections would in general enhance the $\boldsymbol{Q}_{i}$ mass relative to the $q_{i}$ mass. Such enhancement can be substantial. Also, it is possible that a very small but nonzero effective $\bar{\theta}_{c}\left(\leqslant 10^{-8}\right)$ may be introduced by radiative corrections.

As is clear from Eq. (8) and Ref. 10, the axion would not get a mass from $\mathrm{QC}^{\prime} \mathrm{D}$ if the fermions $U$ and/or $D$ are massless. Hence $Q C^{\prime} D$ cannot be 


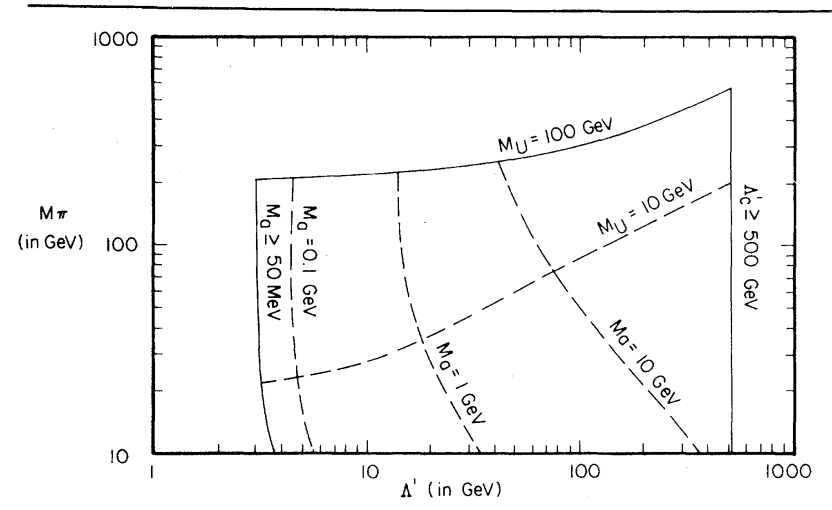

FIG. 1. The $M_{\pi}-\Lambda_{c^{\prime}}$ parameter space expected for the superstrong force $\mathrm{QC}^{\prime} \mathrm{D}$. The axion masses of $m_{a}$ $=50 \mathrm{MeV}, 100 \mathrm{MeV}, 1 \mathrm{GeV}$, and $10 \mathrm{GeV}$ are shown. $M_{U}=M_{D}=100$ and $10 \mathrm{GeV}$ are shown. The lower bound on $M_{\pi}$ is not shown.

identified as the force introduced by Weinberg and Susskind. ${ }^{12}$ In general, in order to have natural $C P$ invariance in QCD there must be an explicit chiral $U(1)$ symmetry left in the Lagrangian after both symmetry breaking and fermion mass generation have taken place. Otherwise, strong $C P$ nonconservation would remain a problem. ${ }^{13}$

To study the phenomenology of $\mathrm{QC}^{\prime} \mathrm{D}$, we must obtain some idea of the ranges of values that $\Lambda_{c^{\prime}}, M_{U, D}$, and $M_{\pi}$ are expected to take. Let us consider the following constraints: (1) If $M_{\pi} \leqslant 18$ $\mathrm{GeV}$, then $e^{+} e^{-} \rightarrow$ " $\pi^{+}$" " $\pi^{-"}$ " could have been observed. The dominant decay mode " $\pi^{+"} \rightarrow l^{+} \nu$, where $l^{+}$is the heaviest lepton permitted by phase space, would provide a clean signal. Also, the dominant decay mode of " $\pi$ " is to two photons. (2) The axion mass must be bigger than (roughly) $50 \mathrm{MeV}{ }^{8}$ This provides a lower bound on $\Lambda_{c^{\prime}}$ $>3 \mathrm{GeV}$ as shown in Fig. 1. (3) The coupling of the $\mathrm{QC}$ 'D pions to the $W^{ \pm}$and $Z^{0}$ bosons contributes a mass term to the gauge bosons as follows: $M_{w}{ }^{2}=\left(g_{w}{ }^{2} / 4\right)\left[f^{2}+F_{\pi}{ }^{2}\right]>g_{w}{ }^{2} F_{\pi}{ }^{2} / 4$, where $g_{w}$ is the weak $\mathrm{SU}(2)$ gauge coupling. This implies that $\Lambda_{c},<500 \mathrm{GeV}$. As $F_{\pi}$ increases and $f$ decreases correspondingly, the axion-fermion coupling strength increases. (4) If the $\mathrm{QC}^{\prime} \mathrm{D}$ fermions are very heavy, they would induce an unacceptably strong $\varphi^{4}$ coupling. ${ }^{14}$ In the absence of fine tuning of the parameters in the Higgs potential, we expect $M_{U, D}<100 \mathrm{GeV}$. This is shown in the figure.

Although some of the constraints mentioned above are not rigorous bounds, they do provide an indication of the ranges of values in which we should expect $\mathrm{QC}^{\prime} \mathrm{D}$ to emerge if it exists. It is encouraging that near-future accelerators can reach essentially the whole allowed parameter space shown in the figure. For small $\Lambda_{c^{\prime}}$, the $\mathrm{QC} \mathrm{C}$ vector meson mass should be comparable to that of the $\mathrm{QC}$ 'D pion mass. Since its dominant couplings are via weak and electromagnetic interactions, it can show up as a resonance in both $e^{+} e^{-}$annihilation and Drell-Yan experiments. Its excited resonance should show up at roughly $2 \Lambda_{c},>6 \mathrm{GeV}$ above it.

I thank W. A. Bardeen, J. D. Bjorken, and M. E. Peskin for discussions. This research was supported in part by the National Science Foundation.

${ }^{1}$ A. A. Belavin, A. M. Polyakov, A。S.Schwartz, and Yu. S. Tyupkin, Phys. Lett. 59B, 85 (1975).

${ }^{2}$ G. 't Hooft, Phys. Rev. Lett. 37, 8 (1976), and Phys. Rev. D 14, 3432 (1976); R. Jackiw and C. Rebbi, Phys. Rev. Lett. 37, 172 (1976); C. G. Callan, R. F. Dashen, and D. J. Gross, Phys. Lett. 63B, 334 (1976).

${ }^{3}$ N. F. Ramsey, Phys. Rep. $\underline{43 \mathrm{C}}, 409$ (1978); V. Baluni, Phys. Rev. D 19, 2227 (1979); R. Crewther, P. DiVecchia, G. Veneziano, and E. Witten, Phys. Lett. 88B, 123 (1979).

${ }^{4}$ R. D. Peccei and H. R. Quinn, Phys. Rev. Lett. 38, 1440 (1977), and Phys. Rev. D 16, 1791 (1977).

${ }^{5}$ S. Weinberg, Phys. Rev. Lett. 19, 1264 (1967); A. Salam, in Elementary Particle Physics, edited by N. Svartholm (Almquist and Wiksells, Stockholm, 1968), p. 367; S. L. Glashow, J. Iliopoulos, and L. Maiani, Phys. Rev. D 2 , 1285 (1970).

${ }^{6}$ S. Weinberg, Phys. Rev. Lett. 40, 223 (1978).

${ }^{7}$ F. Wilczek, Phys. Rev. Lett. 40, 279 (1978).

${ }^{8}$ For a review on experimental bounds, see J. D. Bjorken, unpublished.

${ }^{9}$ For a review on models, see S.-H. H. Tye, Cornell University Report No. CLNS-81/479, 1981 (to be published).

${ }^{10} \mathrm{~W}$. A. Bardeen and S.-H.H. Tye, Phys. Lett. 74B, 229 (1978); we shall follow the notations of this work. See also Ref. 6 and J. Kandaswamy, P. Salomonson, and J.Schechter, Phys. Lett。74B, 377 (1978), and Phys. Rev. D 17, 3051 (1978)。

${ }^{11}$ J. Ellis and M. K. Gaillard, Nucl. Phys. B150, 141 (1979).

${ }^{12}$ S. Weinberg, Phys. Rev. D 19, 1277 (1979); L. Susskind, Phys. Rev. D 20, 2619 (1979).

${ }^{13}$ E. Eichten, K. D. Lane, and J. P. Preskill, Phys. Rev. Lett。 45, 225 (1980); K. Lane, “CP Nonconservation in Dynamically Broken Gauge Theories" (to be published), where earlier references on the subject can be found. In general, strong $C P$ nonconservation is expected in such models of dynamical symmetry breaking as a result of the Dashen mechanism. This would be absent if, after all symmetry breakings and fermion mass generations, there is a remnant chiral $\mathrm{U}(1)$ symmetry which can rotate the remaining $\theta$ angle to zero.

${ }^{14}$ P. Q. Hung, Phys. Rev. Lett. 42, 873 (1979); H. D. Politzer and S. Wolfram, Phys. Lett. 82B, 242 (1979), and 83B, 421(E) (1979). 